\title{
Case Report \\ Superior Ophthalmic Vein Thrombosis with Cerebral Venous Sinus Thrombosis: A Rare Entity in a Child
}

\author{
Kanij Fatema' ${ }^{1}$, Muhammad Mizanur Rahman², Laila Areju Man Banu ${ }^{3}$ \\ Received: March 3, 2019 Accepted: April 25, 2019 \\ doi: https://doi.org/10.3329/jemc.v9i2.41415
}

\begin{abstract}
Cerebral venous sinus thrombosis (CVST) is a rare stroke-like syndrome. Sometimes it may be rarely associated with ophthalmic vein occlusion. We present a 10-year-old girl who had severe headache, diplopia, severe congestion of eye, proptosis and orbital pain. She had no signs of meninigeal irritation, no focal deficit except left sided lateral rectus palsy and altered visual acuity. Investigations regarding vasculitis and thrombophillia were normal except thrombocytosis. MRI of brain showed 'dense triangle sign' and thickened superior ophthalmic vein. Magnetic resonance venography (MRV) showed occlusion of superior sagital sinus and transverse sinus. She was treated with paracetamol, acetazolamide, rivaroxaban and antibiotics followed by high dose pulse methyl prednisolone. She made a partial recovery within one week and at 3 months follow-up she was completely normal.
\end{abstract}

Key words: Superior ophthalmic vein thrombosis; Cerebral venous sinus thrombosis; Rivaroxaban; Acetazolamide

J Enam Med Col 2019; 9(2): 127-132

\section{Introduction}

Cerebral venous sinus thrombosis (CVST) is a rare stroke-like syndrome occurring in only two to five cases per million per year. ${ }^{1}$ It has a significant morbidity and mortality. This entity is associated with hypercoagulable states and can present with simple headaches to localizing neurologic deficits, frank seizures, and coma. Prompt recognition and treatment can prevent significant morbidity and life loss. The risk factors include infection and genetically acquired pro-thrombotic states. ${ }^{2}$ Superior ophthalmic vein thrombosis (SOVT) is another clinical entity which is rare in pediatric population. The diagnosis is both clinical and radiological. It occurs due to congestion of the vein resulting into swelling of eye, proptosis, restriction of eye movement and visual impairment. It occurs due to infectious and noninfectious etiologies. In both cases confirmation of diagnosis is done with MRI and magnetic resonance venography (MRV) of the structures involved. ${ }^{3}$ Here we present a case of a
SOVT and CVST occurring in a 10-year-old girl.

\section{Case report}

A 10-year-old girl presented with headache for 2 months, redness of eye for 2 weeks and fever for 7 days. Prior to this illness she was in good health. Her headache was progressive, tightening in nature, usually more in morning, associated with vomiting which was non-projectile in nature. Later on, she developed redness of eye along with double vision associated with photophobia. She also developed orbital pain but her vision was intact. Her fever was low grade and irregular in nature. She had no history of seizure, loss of consciousness, trauma in head, palpitation, breathlessness, skin rash, photophobia, oral ulcer, contact with tuberculosis patient, joint pain, ear/eye/sinus infection and urinary problem. She was the first issue of non-consanguineous parents and there was no history of migraine or vasculitis in her family.

1. Associate Professor, Department of Pediatric Neurology, Bangabandhu Sheikh Mujib Medical University, Dhaka

2. Professor, Department of Pediatric Neurology, Bangabandhu Sheikh Mujib Medical University, Dhaka

3. Phase-B Student, Department of Pediatric Neurology and Neurodevelopment, Bangabandhu Sheikh Mujib Medical University, Dhaka

Correspondence Kanij Fatema, Email: maiomonami@gmail.com 
The girl was conscious, ill-looking and co-operative. Eyes were congested and proptosis was present bilaterally (Fig 1). She had no pallor, cyanosis, jaundice, clubbing or edema. Anthropometry was within normal range. Vitals were within normal limit. There were no signs of meningeal irritation. Skin survey was normal and BCG mark was present. Ophthalmoscopic examination revealed hyperemic vessels, visual acuity was $6 / 18$ in right eye and 6/12 in left eye unaided. Lateral rectus nerve palsy was present in left side. There was no other neurological deficit in the patient.

Her investigation reports showed $\mathrm{Hb} 12$ gm\%, leukocytosis (total count 13700, neutrophil 80\%), thrombocytosis (total platelet count 6,93000), CRP 12 $\mathrm{mg} / \mathrm{dL}$, normal serum electrolytes and normal liver and renal function tests. Urine for routine examination was normal. PT was 14.9 (control 11.80), INR 1.25, APTT 32 seconds (control 28 seconds). Protein C and Protein $\mathrm{S}$ were normal. Antithrombin III, ANA and Anti-DS DNA were normal. Antiphospholipid antibody was IgG 4.1 U/mL (normal) and anticardiolipin antibody was IgG 5.23 U/L (normal). Homocystine level was 16.36 (high normal) and thyroid function tests were normal. Mantoux test was negative. CSF study revealed normal findings.

Magnetic resonance imaging (MRI) showed the classical 'dense triangle sign' in the posterior part of the superior sagittal sinus which corresponds the fresh thrombus (Fig 3). Thus it suggested thrombosis of superior sagittal sinus. In addition to that there was thickened superior ophthalmic vein with indistinct border due to perivascular edema of both sides. Magnetic resonance venography (MRV) of brain revealed features of dural venous sinus thrombosis involving superior venous sinus and transverse sinus without any evidence of hemorrhagic venous infarct (Fig 4). We diagnosed the case as bilateral ophthalmic vein thrombosis and cerebral venous sinus thrombosis.

As the child presented after two months of onset, heparin was not given. The patient was treated with rivaroxaban (an oral anticoagulant) $10 \mathrm{mg}$ daily. For headache paracetamol was given. Along with this, acetazolamide was also given. As the patient had fever with increased WBC count, injectable antiobiotic was given for 7 days. Her headache decreased. But congestion of eye was still there along with lateral rectus palsy. Methylprednisolone was started and continued for five days. After one dose of steroid, there was dramatic improvement of orbital pain and congestion of eye (Fig 2). After three months the girl was completely normal without any residual focal deficit, eye abnormalities or headache.

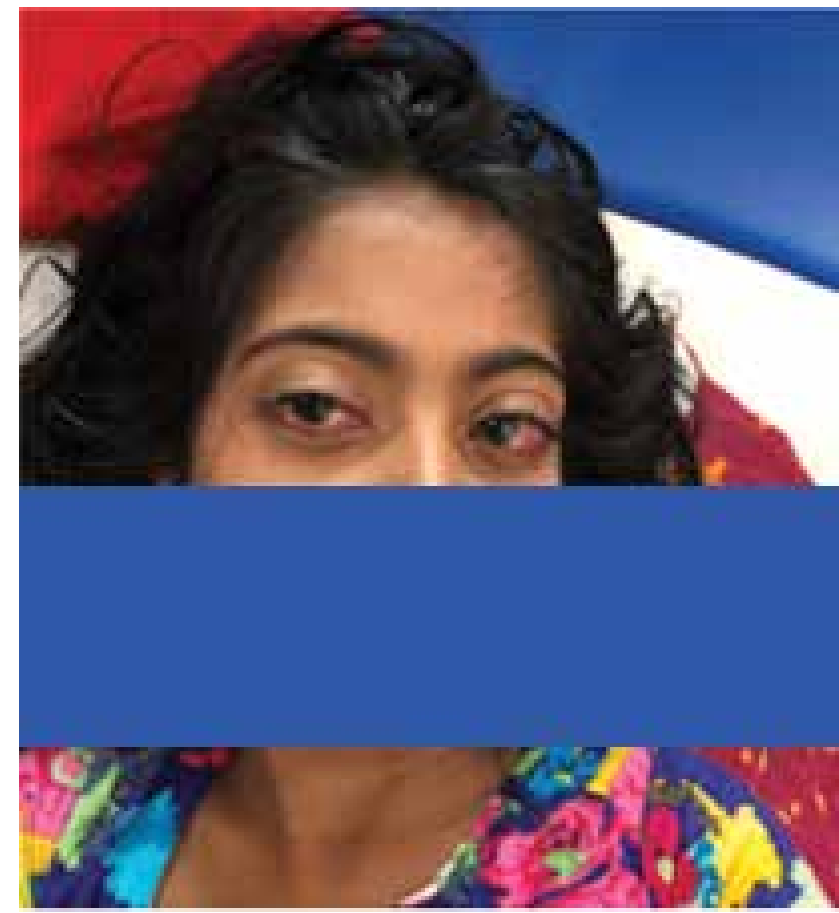

Fig 1. Congestion of eye and lateral rectus palsy in a girl with CVST and SOVT

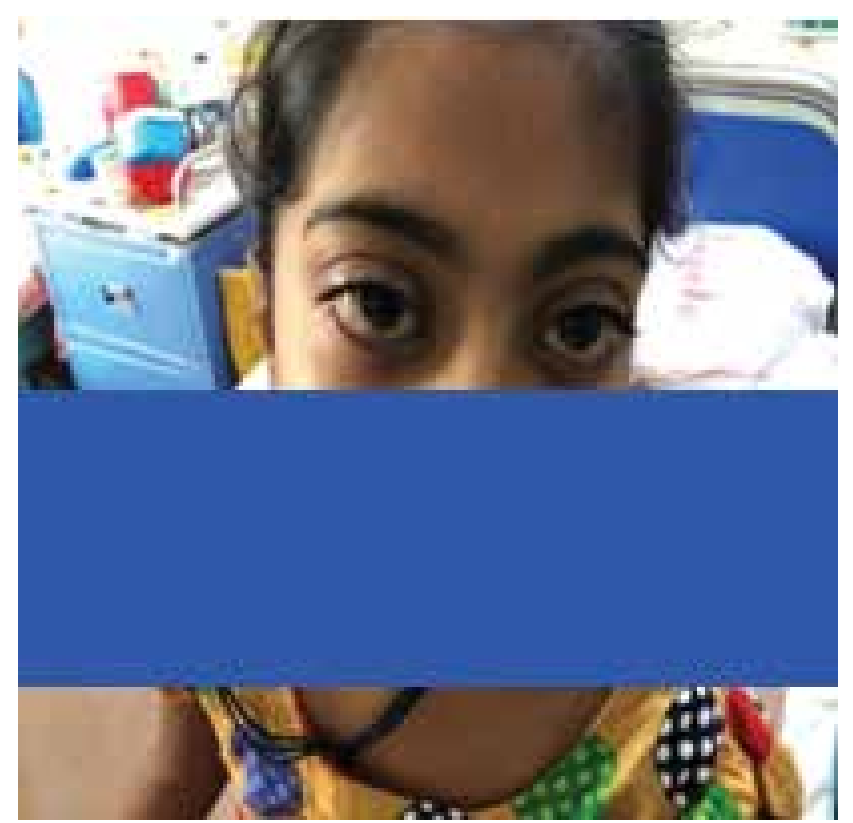

Fig 2. Improvement of congestion after pulse methylprednisolone 


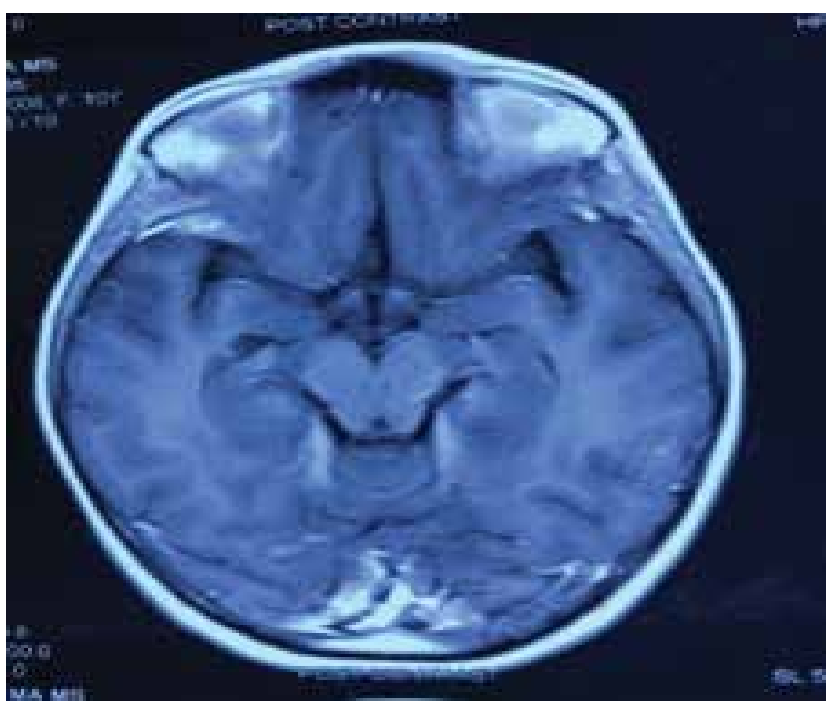

Fig 3. MRI of brain with contrast showing 'dense triangle sign'

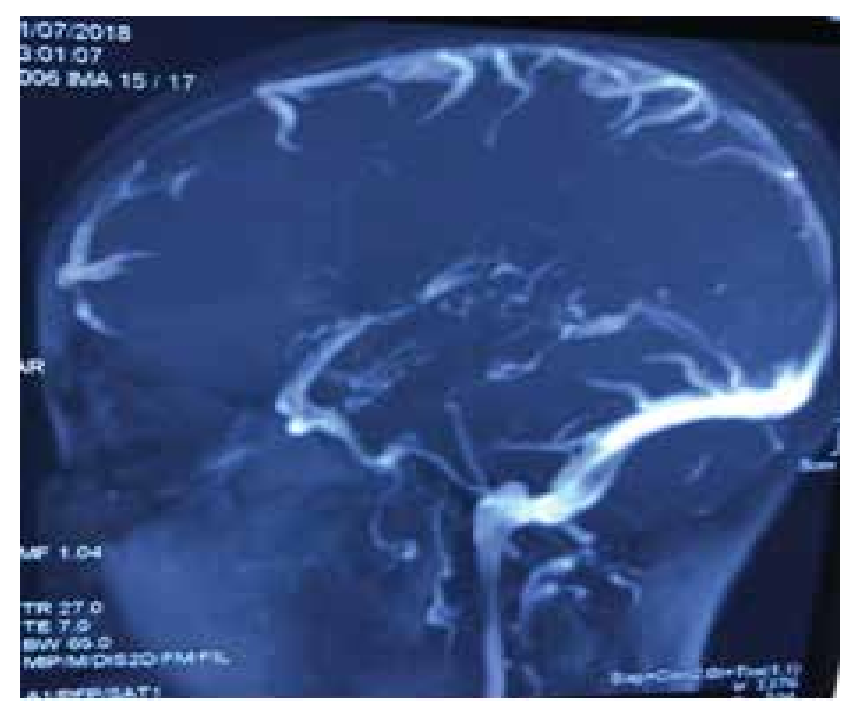

Fig 4. MRV of brain showing filling defect in superior sagittal sinus and transverse sinus

\section{Discussion}

CVST is an uncommon type of stroke, which may account up to 0.5 to $1 \%$ of all strokes. ${ }^{4}$ Although CVST has been reported in adults, it has more commonly been observed in neonates and children in hospital-based studies. ${ }^{2,5}$ The ratio of adult females to males is $3: 1{ }^{2,6}$ It can create diagnostic dilemma as the presentation is widely variable including isolated headache, mood changes, cranial nerve palsies, ocular complaints, aphasia, encephalopathy, TIA/stroke symptoms, seizure or frank coma. Headache is the most consistent complaint and is classically described as slow onset, dull, localized and worse with recumbent position. ${ }^{2}$

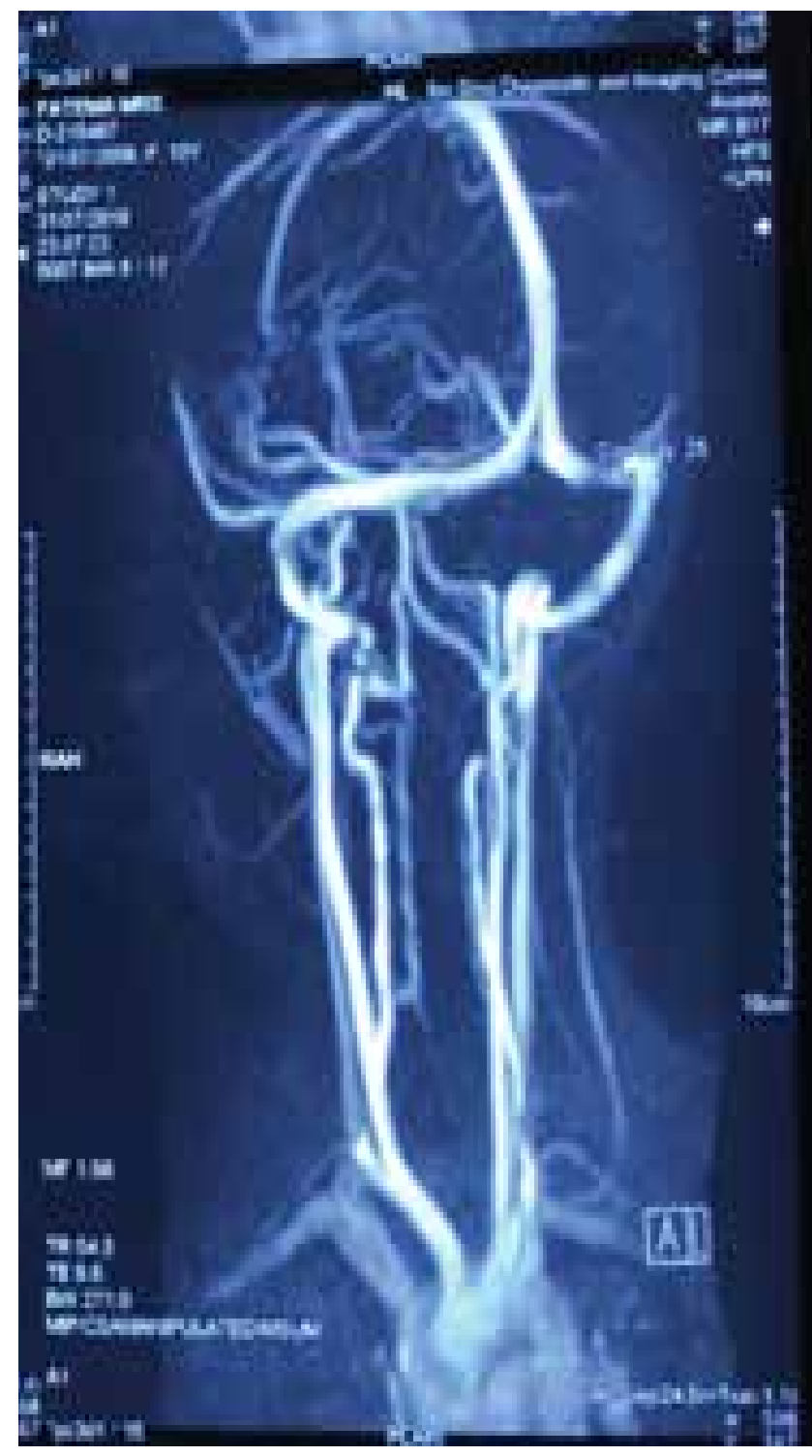

Fig 5. MRV of brain showing defect in superior sagittal sinus, transverse sinus and internal jugular veins

Traditionally CVST is of two types - septic and aseptic. Risk factors related to CVST are diverse. At least one risk factor could be identified in more than $85 \%$ of patients with CVST. $^{7}$ The common risk factors are pregnancy and puerperium, drugs like oral contraceptives, steroid, L-asparginase, hormone replacements, malignancy, systemic inflammatory disease like systemic lupus erythematosus, infectious diseases, hematological disorders like polycythemia, dehydration, coagulation disorders, congenital heart diseases, head trauma etc. ${ }^{8}$ In this case patient had leukocytosis and thrombocytosis. These may be the risk factors in this case. All other blood tests and urine 
investigation were within normal limits. Based on Bousser, four clinical patterns for CVST have been identified - a) Focal syndrome: presence of focal signs associated with headache, seizures or changes in mental state, b) Isolated intracranial hypertension (ICH): headache, nausea, vomiting and papilledema, c) Diffuse subacute encephalopathy: changes in mental state and d) Cavernous sinus syndrome: painful ophthalmoplegia, chemosis and proptosis. ${ }^{9}$ Our case matches with the type 4, but here there was evidence of left cavernous sinus thrombosis (left $6^{\text {th }}$ nerve palsy).

SOVT is usually found in cases of orbital congestion such as orbital cellulitis, idiopathic orbital inflammation, vascular malformation, infection, trauma, tumor and coagulopathy. ${ }^{10,11}$ Some systemic diseases have been reported in association with SOVT like systemic lupus erythematosus, Graves disease, ulcerative colitis etc. ${ }^{12-16}$ Other causes are hematologic like antiphospholipid syndrome, sickle cell disease, hormonal causes like tamoxifen, oral contraceptive pills etc, Tolosa-Hunt syndrome, idiopathic orbital inflammatory disease. ${ }^{17-25}$ In a case series by Nicolien et $\mathrm{al}^{3}$ the majority of the patients were aseptic (seven out of nine) although all the patients in this case series were adults. The septic causes were sinusitis, dental, orbital and facial infection. The underlying cause in our case was CVST. The presenting symptoms are swelling of eyes, chemosis, proptosis, impaired eyelid motility, reduced visual acuity and increased intraocular pressure. ${ }^{3}$ In our case we found proptosis, chemosis, impaired visual acuity and restricted motility of eyeball although here intraocular pressure was normal. The clinical presentations may be explained by congestion of the orbit due to impaired venous drainage. This mechanism is also present in CVST, thus both SOVT and CVST may occur together like in our case. In our case the SOVT was bilateral but in literature review most of the cases of SOVT found are unilateral. ${ }^{3}$

The main modality to confirm the diagnosis both CVST and SOVT is neuroimaging. Various imaging techniques are used; CT angiography and MRI/MRV being most sensitive and specific. In CVST the 'dense triangle sign' is a hyperdense triangular or round shape in the posterior superior sagittal sinus that has been described in non-contrast head CT scans. 'Empty delta sign' seen with contrast-enhanced CT scan is a triangular ring of enhancement with a central region lacking contrast in the posterior superior sagittal sinus. ${ }^{26}$ In case of our patient, MRI showed the classical 'dense triangle sign' in the posterior part of the superior sagittal sinus which corresponds to fresh thrombus. Her MRV showed non-visualized superior sagittal sinus and transverse sinuses which confirmed the diagnosis.

The diagnosis of SOVT is confirmed preferably by contrast-enhanced CT or MR imaging. On contrastenhanced CT scan SOVT is characterized by a thickened ophthalmic vein with irregular border. MRI is the modality of choice for confirming SOVT as it shows all stages of thrombus formation. Here a filling defect can be seen within the SOV itself, or a thin lining can be present close to the vessel wall, either due to vessel wall enhancement or a small lumen in between the clot and the vessel wall. ${ }^{27,28}$ In case of a chronic SOV thrombosis, the central thrombus can also show enhancement opposite to the acute stage. MRI shows enhancement of intraorbital fat and swelling and enhancement of the eye musculature due to venous congestion. Similar to contrast enhanced CT, MRI can show intra-luminal filling defects in the SOV. Secondary signs include proptosis and thickening of the extraocular muscles. ${ }^{29}$ MRI also excludes carotidcavernous sinus fistula, cavernous sinus thrombosis and sino-orbital infection. ${ }^{17}$ Orbital color Doppler imaging also allows noninvasive confirmation of SOVT ${ }^{28}$ In our case we have done MRI of brain with contrast with MRV which revealed thickened bilateral SOV with irregular border.

In CVST anticoagulant in the form of heparin in acute stage and warfarin in maintenance phase are suggested if there is no hemorrhage or contraindication. In case of transient risk factor such as infection, trauma or pregnancy, a treatment of three months is recommended while in prothrombotic states a prolonged duration of treatment is suggested. No definite data are there about the duration. However, mostly 6-12 months treatment is given. ${ }^{31}$ As our patient presented late, we prescribed a substitute of warfarin, rivaroxaban which needed minimal monitoring. Addressing the etiology, as our patient had leukocytosis without definite focus of infection, we gave the patient a short course of injectable antibiotic. As there was severe headache initially, we gave her paracetamol and acetazolamide anticipating increased 
intracranial pressure. For SOVT antibiotics, steroids and anticoagulants are the mainstay of treatment. Surgery may be needed for an underlying sinusitis, orbital abscess or dental infection. ${ }^{3}$ Our patient had severe congestion of both eyes, thus we treated the patient with pulse methylprednisolone which caused dramatic improvement of the congestion and orbital pain.

Early detection and treatment and identification of risk factor are very important for favorable outcome in both CVST and SOVT. Most patients experience complete resolution over several weeks with treatment. Our patient improved in hospital with treatment and at three month follow-up she was completely normal without any residual clinical feature.

\section{Conclusion}

Our case had some exceptional criteria found rarely - pediatric onset, SOVT coexisting with CVST and dramatic response to steroid and anticoagulant. The underlying cause was thrombocytosis which is nonspecific. Thus in a pediatric patient with new onset severe headache along with proptosis and congestion of eye a suspicion of SOVT and CVST can be made as early diagnosis has positive implication in management and prognosis.

\section{References}

1. Devasagayam S, Wyatt B, Leyden J, Kleinig $\mathrm{T}$. Cerebral venous sinus thrombosis incidence is higher than previously thought. Stroke 2016; 47: 2180-2182.

2. Ferro JM, Canhão $P$, Stam J, Bousser MG, Barinagarrementeria $\mathrm{F} \&$ ISCVT Investigators. Prognosis of cerebral vein and dural sinus thrombosis: results of the International Study on Cerebral Vein and Dural Sinus Thrombosis (ISCVT). Stroke 2004; 35: 664-670.

3. Nicolien A, Van der P, Kornelis DW, Berg RV, Win MM, Mourits MP. Impact of superior ophthalmic vein thrombosis: a case series and literature review. Orbit 2018; 24: 1-7.

4. Saposnik G, Barinagarrementeria F, Brown RD, Bushnell CD, Cucchiara B, Cushman $M$ et al. Diagnosis and management of cerebral venous thrombosis. A statement for healthcare professionals from the American heart association/American stroke association. Stroke 201: 42, 1158-1192.
5. Lancon JA, Killough KR, Tibbs RE, Lewis AI, Parent AD. Spontaneous dural sinus thrombosis in children. Pediatr Neurosurg 1999; 30: 23-29.

6. Coutinho JM, Ferro JM, Canhão P, Barinagarrementeria F, Cantú C, Bousser MG et al. Cerebral venous and sinus thrombosis in women. Stroke 2009; 40: 2356 $-2361$

7. Piazza G. Cerebral venous thrombosis. Circulation 2012; 125: 1704-1709.

8. Ehtisham A, Stern BJ. Cerebral venous thrombosis: a review. Neurologist 2006; 12: 32-38.

9. Bousser MG, Ferro JM. Cerebral venous thrombosis: an update. Lancet Neurol 2007; 6: 162-170.

10. Berenholz L, Kessler A, Shlomkovitz N, Sarfati S, Segal S. Superior ophthalmic vein thrombosis: complication of ethmoidal rhinosinusitis. Arch Otolaryngol Head Neck Surg 1998; 124: 95-97.

11. Ghosheh FR, Kathuria SS. Intraorbital heroin injection resulting in orbital cellulitis and superior ophthalmic vein thrombosis. Ophthal Plast Reconstr Surg 2006; 22: 473-475.

12. Sorrentino D, Taubenslag KJ, Bodily LM, Duncan K, Stefko T, Yu JY. Superior ophthalmic vein thrombosis: a rare complication of Graves' orbitopathy. Orbit 2018; 37(3): 175-178.

13. Park HS, Gye HJ, Kim JM, Lee YJ. A patient with branch retinal vein occlusion accompanied by superiorophthalmic vein thrombosis due to severe superior ophthalmic vein enlargement in a patient with graves ophthalmopathy. J Craniofac Surg 2014; 25(4): e322-324.

14. Baidoun F, Issa R, Ali R, Al-Turk B. Acute unilateral blindness from superior ophthalmic vein thrombosis: rare presentation of nephrotic syndrome from class IV lupus nephritis in the absence of antiphospholipid or anticardiolipin syndrome. Case Rep Hematol 2015: Article ID 413975. Available at: http://dx.doi. org/10.1155/2015/41397. Accessed January 2019.

15. Sambhav K, Shakir O, Chalam KV. Bilateral isolated concurrent superior ophthalmic vein thrombosis in systemic lupus erythematosus. Int Med Case Rep J 2015; 8: 181-183.

16. Mandic JJ, Mandic K, Mrazovac D. Superior ophthalmic vein thrombosis with complete loss of 
vision as a complication of autoimmune and infective conditions. Ocul Immunol Inflamm 2017; 26(7):10661068.

17. Lim LH, Scawn RL, Whipple KM, Oh SR, Lucarelli MJ, Korn BS et al. Spontaneous superior ophthalmic vein thrombosis: a rare entity with potentially devastating consequences. Eye 2014; 28(3): 348-351.

18. Dey M, Charles Bates A, McMillan P. Superior ophthalmic vein thrombosis as an initial manifestation of antiphospholipid syndrome. Orbit 2013; 32(1): 4244.

19. Idrees Z, Dooley I, Jackson A, Roche C, Fahy G. Simultaneous isolated bilateral superior orbital vein thrombosis as a presenting feature of antiphospholipid syndrome. Orbit 2014; 33(3): 214-216.

20. Rao R, Ali Y, Nagesh CP, Nair U. Unilateral isolated superior ophthalmic vein thrombosis. Indian $\mathrm{J}$ Ophthalmol 2018; 66(1): 155-157.

21. Lai PF, Cusimano MD. The spectrum of cavernous sinus and orbital venous thrombosis: a case and a review. Skull Base Surg 1996; 6: 53-59.

22. Michaelides $\mathrm{M}$, Aclimandos W. Bilateral superior ophthalmic vein thrombosis in a young woman. Acta Ophthalmol Scand 2003; 81: 88-90.

23. Jaais F, Habib ZA. Unilateral superior ophthalmic vein thrombosis in a user of oral contraceptives. Med
J Malaysia 1994; 49: 416-418.

24. Spirn FH, Wolintz AH, Tenner MS, Gombos GM. Tolosa hunt syndrome. Ann Ophthalmol 1975; 7: 1087-1090.

25. Carrim ZI, Ahmed TY, Wykes WN. Isolated superior ophthalmic vein thrombosis with orbital congestion: a variant of idiopathic orbital inflammatory disease? Eye 2007; 21(5): 665-666.

26. Ferro JM, Canhão P. Etiology, clinical features, and diagnosis of cerebral venous thrombosis. Available at: https://www.uptodate.com. Accessed August 2018.

27. Walker JC, Sandhu A, Pietris G. Septic superior ophthalmic vein thrombosis. Clin Exp Ophthalmol 2002 ; 30: 144-146.

28. Vyas S, Das PJ, Gupta SK, Kakkar N, Khandelwal N. Superior and inferior ophthalmic veins thrombosis with cavernous sinus meningioma. Middle East African Journal of Ophthalmology 2011; 18(3): 256258 .

29. Coban G, Cetinkaya A, Karalezli A, Donmez FY, Ozbek N. Unilateral superior ophthalmic vein thrombosis in a neonate. Ophthal Plast Reconstr Surg 2013; 29(6): e154-156.

30. Guenther G, Arauz A. Cerebral venous thrombosis: a diagnostic and treatment update. Neurología 2011; 26(8): 488-498. 